\title{
HÖLDER-CONTINUITY OF THE SOLUTIONS FOR OPERATORS WHICH ARE A SUM OF SQUARES OF VECTOR FIELDS PLUS A POTENTIAL
}

\author{
G. CITTI AND G. DI FAZIO
}

(Communicated by Barbara L. Keyfitz)

\begin{abstract}
In this paper we study the local Hölder-regularity of weak solutions to $\mathscr{L} u+V u=0$ where $\mathscr{L}$ is a Hörmander hypoelliptic operator and the potential $V$ belongs to a new class of functions which is the natural extension of Morrey spaces to this situation. We improve a recent result of Citti, Garofalo, and Lanconelli.
\end{abstract}

\section{INTRODUCTION}

The regularity properties of solutions of the Schrödinger equation

$$
\mathscr{L} u+V u=0 \text { in an open bounded subset of } \Omega \text { of } R^{n}
$$

has been intensively studied in the last few years.

When $\mathscr{L}$ is a second-order uniformly elliptic operator in divergence form, Hölder regularity was initially obtained if $V \in L^{p}(\Omega), p>n / 2$. This condition on $V$ is the best possible in the scale of $L^{p}$ spaces (see [LU]) but, in general, it is possible to weaken it. Indeed in 1982 Aizenman and Simon (see [AS]) proved Harnack's inequality for positive solutions of (1) assuming ( $L=\Delta$ and) $V$ in the Stummel-Kato class $S(\Omega)$, i.e., assuming that

$$
\lim _{r \rightarrow 0}\left(\sup _{x \in \Omega} \int_{|x-y|<r} \frac{|V(y)|}{|x-y|^{n-2}} d y\right)=0 .
$$

Let us explicitly note that Aizenman and Simon's result improve the previous one in [S], since $L^{p}(\Omega) \subset S(\Omega)$ for every $p>n / 2$ and $\bigcup_{p>n / 2} L^{p}(\Omega) \neq S(\Omega)$.

The proof in [AS] relies on some probabilistic techniques. However, in 1986, Chiarenza, Fabes, and Garofalo in [CFG], gave a new, nonprobabilistic proof of Harnack's inequality and obtained local continuity of weak solutions of equation (1) (see also Hinz and Kalf [HK] and Simader [Si] for analogous results with $\mathscr{L}=\Delta$ ). These solutions are in general not Hölder continuous, but they have this regularity when $V$ belongs to a particular subset of $S(\Omega)$, the classical

Received by the editors April 29, 1992 and, in revised form, February 9, 1993.

1991 Mathematics Subject Classification. Primary 35B45, 35B60, 35H05, 22 E30. 
Morrey space $L^{1, \lambda}(\Omega)$ for $\lambda>1$ (see [D]). $V$ belongs to this space when

$$
\sup _{\substack{x \in \Omega \\ r>0}} r^{-\mu} \int_{|x-y|<r}|V(y)| d y<+\infty, \quad \mu=\lambda(n-2) .
$$

More recently the same question was investigated for operators $\mathscr{L}$ which are the sum of squares of vector fields. More precisely let $\mathscr{L}$ be an operator of the form

$$
\mathscr{L} \equiv \sum_{j=1}^{p} X_{j}^{2}
$$

where $X_{1}, \ldots, X_{p}$ are $C^{\infty}$ first-order vector fields on an open set $\Omega \subset R^{n}$ satisfying Hörmander's condition for hypoellipticity

$$
\operatorname{rank} \operatorname{Lie}\left[X_{1}, \ldots, X_{p}\right](x)=n
$$

at every point $x \in \Omega$ and such that

$$
X_{j}=X_{j}^{*} \text { for every } j=1, \ldots, p
$$

( $X_{j}^{*}$ is the formal adjoint of $X_{j}$ ).

In [CGL], assuming $V$ to be in an appropriate version of the Stummel-Kato class defined below, a Harnack inequality and local continuity of weak solutions of (1) were established.

In this paper, assuming again that $\mathscr{L}$ satisfies (3)-(5), we introduce a new space $L_{\mathscr{L}}^{1, \lambda}(\Omega)$, which is the natural generalization of the classical Morrey space to the framework of these operators and which contains the $\mathscr{L}$-StummelKato class introduced in [CGL]. In $\S 3$ we list some examples for the reader's convenience. Then, in $\S 4$ we extend the results in [D] to weak solutions of $\mathscr{L} u+V u=0$, proving their local Hölder continuity when the potential $V$ belongs to $L_{\mathscr{L}}^{1, \lambda}(\Omega)$.

The authors take this opportunity to thank E. Lanconelli for many helpful conversations about this work.

\section{DEFINITION OF MORREY SPACES}

In the sequel we assume that the operator $\mathscr{L}$ satisfies (3)-(5) and coincides with the Laplacian $\Delta$ in the complement of some compact subset of $\Omega$. This condition does not imply any restriction because of the local character of our result. On the other side it ensures the existence of a fundamental solution $\Gamma$ of class $C^{\infty}$ off the diagonal of $R^{n} \times R^{n}$ (see [S]). We will call $\Omega_{r}$ its level sets, which are the natural analogues of the Euclidean balls for the Laplace operator:

$$
\Omega_{r}(x) \equiv\left\{y \in \Omega: \frac{1}{\Gamma(x, y)}<r\right\} \quad \text { with } \quad r>0 \text { and } x \in \Omega .
$$

Let $\eta: R^{+} \rightarrow R^{+}$be an increasing function such that $\lim _{r \rightarrow 0} \eta(r)=0$.

Recall that a function $V \in L_{\text {loc }}^{1}(\Omega)$ belongs to the $\mathscr{L}$-Stummel-Kato class $S(\Omega)$ when there exists a constant $C>0$ such that

$$
\sup _{x \in \Omega} \int_{\Omega_{r}(x)}|V(y)| \Gamma(x, y) d y \leq C \eta(r) .
$$

In particular, when $\eta(r)=r^{\lambda}$, we will simply denote $S_{\lambda}(\Omega)=S_{r^{\lambda-1}}(\Omega)$. Now we give our definition of $\mathscr{L}$-Morrey spaces. 
Definition 2.1. If $\lambda>1$, we will say that $V$ belongs to the Morrey space $L_{\mathscr{L}}^{1, \lambda}(\Omega)$, if $V \in L_{\text {loc }}^{1}(\Omega)$ and

$$
\|V\|_{L_{\mathscr{L}}^{1, \lambda}(\Omega)} \equiv \sup _{\substack{r>0 \\ x \in \Omega}} r^{-\lambda} \int_{\Omega_{r}(x)}|V(y)| d y<+\infty .
$$

Remark 2.1. We explicitly note that in the case $\mathscr{L}=\Delta, L_{\Delta}^{1, \lambda}$ is the classical Morrey space $L^{1, \lambda(n-2)}$.

The following relation holds between these spaces.

Proposition 2.1. If $\lambda>1$ then $L_{\mathscr{L}}^{1, \lambda}(\Omega)=S_{\lambda}(\Omega)$.

Proof. Let $V \in L_{\mathscr{L}}^{1, \lambda}(\Omega)$. Then for every $x \in \Omega$ and $r>0$, we have

$$
\begin{aligned}
\int_{\Omega_{r}(x)} & |V(y)| \Gamma(x, y) d y=\sum_{k=0}^{\infty} \int_{\Omega_{r(x) / 2^{k}} \backslash \Omega_{r(x) / 2^{k+1}}}|V(y)| \Gamma(x, y) d y \\
\leq & \sum_{k=0}^{\infty} \frac{r}{2^{k+1}} \int_{\Omega_{r(x) / 2^{k}}}|V(y)| d y \leq 2 \sum_{k=0}^{\infty} 2^{k(1-\lambda)}\|V\|_{L_{\mathscr{L}}^{1}(\Omega)} r^{\lambda-1} \leq\|V\|_{L_{\mathscr{L}^{1}}^{1, \lambda}(\Omega)} r^{\lambda-1} .
\end{aligned}
$$

Hence we get $L_{\mathscr{L}}^{1, \lambda}(\Omega) \subseteq S_{\lambda}(\Omega)$. The other inclusion is obvious, since

$$
r^{-\lambda} \int_{\Omega_{r}(x)}|V(y)| d y \leq r^{-\lambda+1} \int_{\Omega_{r}(x)} \Gamma(x, y)|V(y)| d y .
$$

\section{EXAMPLES}

Now we give some examples to clarify our definition.

Example 3.1. Sublaplacian operators on homogeneous spaces. Let $\mathscr{L}$ be an operator satisfying (3)-(5). If the Lie algebra $g=\operatorname{Lie}\left(X_{1}, \ldots, X_{p}\right)$ is nilpotent and has dimension $n$, it is possible to define a group law on $R^{n}$, which makes $R^{n}$ into a Lie group, $G$. Its Lie algebra is $g$, and the exponential map is merely the identity. We say that $g$ is stratified and $\mathscr{L}$ is a sublaplacian for $G$ if there exists $m$ such that

$$
g=\bigoplus_{i=1}^{m} V_{j}
$$

where $V_{1}=\left\langle X_{1}, \ldots, X_{p}\right\rangle, V_{j+1}=\left[V_{j}, V_{1}\right]$ for every $j=1, \ldots, m-1$, and $\left[V_{m}, V_{1}\right]=0$. In this case a natural family of algebra automorphisms for the operator $\mathscr{L}$ is

$$
\delta_{r}\left(\sum_{j=1}^{m} Y_{j}\right)=\sum_{j=1}^{m} r^{j} Y_{j} \quad\left(Y_{j} \in V_{j}\right) .
$$

In other words it is possible to choose a basis in $R^{n}$ such that every element $x$ can be represented in the form $x=\left(x_{1}, \ldots, x_{m}\right)$, with $x_{1} \in R^{\operatorname{dim} V_{1}}, \ldots, x_{m} \in$ $R^{\operatorname{dim} V_{m}}$ and $\delta_{r}$ is simply

$$
\delta_{r}\left(x_{1}, \ldots, x_{m}\right)=\left(r x_{1}, \ldots, r^{m} x_{m}\right)
$$


On $R^{n}$ we can also define the function

$$
d_{0}\left(x_{1}, \ldots, x_{m}\right)=\left(\sum_{j=1}^{m}\left|x_{j}\right|^{2 m / j}\right)^{1 / 2 m} .
$$

This is a norm, which is homogeneous of degree one with respect to the dilations $\delta_{r}$ and satisfies

$$
d_{0}(x)=0 \text { if and only if } x=0 ; d_{0}\left(x^{-1}\right)=d_{0}(x) .
$$

(Here $x^{-1}$ denotes the inverse element in the group law defined on $R^{n}$, while the group law itself will be denoted o.) Thus the function

$$
d(x, y)=d_{0}\left(x^{-1} \circ y\right)
$$

is a distance on $R^{n}$, called the control distance associated to $\mathscr{L}$. The real number $Q=\sum_{j=1}^{m} j \operatorname{dim}\left(V_{j}\right)$ is called the homogeneous dimension of $G$ on analogy with the Euclidean dimension. Indeed, since $\delta_{r}$ sends $B(x, 1)$ to $B(x, r)$ for every $x \in R^{n}$ and $r>0$, then a simple change of variable gives

$$
|B(x, r)|=r^{Q}|B(x, 1)|,
$$

where $|\cdot|$ is Lebesgue measure. Moreover, a precise estimate of $\Gamma$ is known (see $[\mathrm{F}])$ :

$$
\Gamma(x, y) \cong d(x, y)^{-Q+2} .
$$

Hence Definition 2.1 can be rewritten in the following way: the function $V$ belongs to $L_{\mathscr{L}}^{1, \lambda}(\Omega)$ if

$$
\sup _{\substack{x \in \Omega \\ r>0}} r^{-\mu} \int_{d(x, y)<r}|V(y)| d y<+\infty, \quad Q-2<\mu
$$

where $\mu=\lambda(Q-2)$.

Examples of sublaplacians follow.

Example 3.2. The classical Laplacian. If $\mathscr{L}=\Delta$ then by (6)

$$
g=\operatorname{Lie}\left(X_{1}, \ldots, X_{n}\right)=\left\langle X_{1}, \ldots, X_{n}\right\rangle .
$$

The dilations in (7) simply reduce to $\delta_{r}(x)=r x$, and the homogeneous dimension is $Q=n$. Similarly from (8) we deduce that the natural distance is the Euclidean one and the definition of $L_{\mathscr{L}}^{1, \lambda}$ is simply the classical definition of Morrey space $L^{1, \lambda(n-2)}(\Omega)$.

Example 3.3. The sublaplacian operator on the Heisenberg group. Let us denote by $\xi=(x, y, t)$ the elements of $R^{2 n+1}$, with $x \in R^{n}, y \in R^{n}, t \in R$, and set

$$
X_{j}=\frac{\partial}{\partial x_{j}}+2 y_{j} \frac{\partial}{\partial t}, \quad Y_{j}=\frac{\partial}{\partial y_{j}}-2 x_{j} \frac{\partial}{\partial t},
$$

and

$$
\mathscr{L}=\sum_{j=1}^{n} X_{j}^{2}+Y_{j}^{2}
$$


The Lie algebra associated has rank $Q=2 n+2$, and the group $G$ is $R^{2 n+1}$ with the group law

$$
(x, y, t) \circ\left(x^{\prime}, y^{\prime}, t^{\prime}\right)=\left(x+x^{\prime}, y+y^{\prime}, t+t^{\prime}+2\left(x y^{\prime}-x^{\prime} y\right)\right) .
$$

Moreover, $\mathscr{L}$ is a sublaplacian operator on $G$. Indeed $g=V_{1} \oplus V_{2}$, where

$$
V_{1}=\left\langle X_{1}, \ldots, X_{n}, Y_{1}, \ldots, Y_{n}\right\rangle, \quad V_{2}=\left\langle\frac{\partial}{\partial t}\right\rangle .
$$

Thus $Q=\operatorname{dim} V_{1}+2 \operatorname{dim} V_{2}=2 n+2$, the dilations are

$$
\delta_{\lambda}(x, y, t)=\left(\lambda x, \lambda y, \lambda^{2} t\right),
$$

and the homogeneous norm is

$$
d_{0}(x, y, t)=\left(\left(x^{2}+y^{2}\right)^{2}+t^{2}\right)^{1 / 4} .
$$

Remark 3.1. For a general operator satisfying (3)-(5) the homogeneous dimension is not defined, but it is possible to define a local homogeneous dimension, which, however, cannot be used to give a definition of Morrey space analogous to (9). For the reader's convenience we recall the definition.

Let $U$ be a bounded open set such that $U \subset \subset \Omega$. If $X_{0}, \ldots, X_{p}$ together with their commutators of length at most $m$ span $R^{n}$ at every point of $U$, we say that $X_{0}, \ldots, X_{p}$ are of type $m$, and we set

$$
X^{(1)}=\left\{X_{0}, \ldots, X_{p}\right\}, X^{(2)}=\left\{\left[X_{0}, X_{1}\right], \ldots,\left[X_{p-1}, X_{p}\right]\right\} \text {, etc. }
$$

Let $Y_{1}, \ldots, Y_{q}$ be some enumeration of the components of $X^{(1)}, \ldots, X^{(m)}$. If $Y_{i} \in X^{(j)}$, we say that $Y_{i}$ has formal degree $\operatorname{deg}_{i}=\operatorname{deg}\left(Y_{i}\right)=j$. If $I=$ $\left(i_{1}, \ldots, i_{n}\right) \in N^{n}$, with $1 \leq i_{j} \leq q$ and $x \in U$, we set

$$
\operatorname{deg}(I)=\operatorname{deg}_{i_{1}}+\cdots+\operatorname{deg}_{i_{n}}, \quad \lambda_{I}(x)=\operatorname{det}\left(Y_{i_{1}}, \ldots, Y_{i_{n}}\right) .
$$

We also call, following [NSW]

$$
\Lambda(x, r)=\sum_{I}\left|\lambda_{I}(x)\right| r^{\operatorname{deg}(I)} \quad \forall x \in U, r>0,
$$

where the index $I$ in the summation varies in the set of all $n$-uple previously defined. For $\delta>0$, let $C(\delta)$ be the class of absolutely continuous mappings $\varphi:[0,1] \rightarrow \Omega$ such that $\varphi^{\prime}(t)=\sum_{j=1}^{q} a_{j}(t) Y_{j}(\varphi(t))$ a.e. $t \in[0,1]$ with $\left|a_{j}(t)\right|<\delta^{d_{j}}$. Then define

$$
d(x, y)=\inf \{\delta>0: \exists \varphi \in C(\delta) \text { with } \varphi(0)=x, \varphi(1)=y\} .
$$

The function $d$ defines a metric, and $\Lambda$ gives an estimate of the Lebesgue measure of the ball of this metric. Indeed in [NSW] and [SC] the following results are proved.

For every bounded set $U \subset \subset \Omega$ there exist $C_{1}, C_{2}, r_{0}>0$ such that, for every $x \in U, r<r_{0}$,

$$
C_{1} \leq \frac{|B(x, r)|}{\Lambda(x, r)} \leq C_{2}
$$

where $|\cdot|$ stands for Lebesgue measure. 
For every bounded set $U \subset \subset \Omega$ there exist $C_{1}, C_{2}, r_{0}>0$ such that for every $x \in U$ and every $y \in U \backslash\{x\}$ with $d(x, y) \leq r_{0}$

$$
C_{1} \frac{d(x, y)^{2}}{|B(x, d(x, y))|} \leq \Gamma(x, y) \leq C_{2} \frac{d(x, y)^{2}}{|B(x, d(x, y))|} .
$$

From (11) it follows that the following limit exists:

$$
Q(x)=\lim _{r \rightarrow 0^{+}} \frac{\log (|B(x, r)|)}{\log r} .
$$

Here $Q(x)$ is called the local homogeneous dimension, and when $\mathscr{L}$ is a sublaplacian operator, $Q(x)$ is the homogeneous dimension.

The following example will clarify the meaning by Definition 2.1 when the local homogeneous dimension is not a constant.

Example 3.4. The Grushin operator. Let us denote by $\xi=(x, y)$ the elements of $R^{n}$ with $x \in R^{M}, y \in R^{N}$ and $n=M+N$. Then the Grushin operator is defined as

$$
\mathscr{L} \equiv \sum_{j=1}^{M} X_{j}^{2}+\sum_{j=1}^{N} Y_{j}^{2}
$$

where

$$
X_{j}=\frac{\partial}{\partial x_{j}} \quad \text { and } \quad Y_{j}=x_{j} \frac{\partial}{\partial y_{j}} .
$$

Since the rank of the Lie algebra $g$ generated by the vector fields $X_{j}$ and $Y_{j}$ is $M+2 N$ and the rank of the image of the same Lie algebra at every point is $M+N, \mathscr{L}$ is not a sublaplacian operator. Its local homogeneous dimension is

$$
Q(x, y)= \begin{cases}M+N, & x \neq 0, y \in R^{N}, \\ M+2 N, & x=0, y \in R^{N} .\end{cases}
$$

Let us now note that the class defined by (9) contains only the null function. Indeed, if $\Omega$ is an open set containing the origin and $\mu$ is a real number greater than $Q(x, y)-2$ for all $(x, y) \in \Omega$, then $\mu$ is larger than $M+2 N-2$. Thus if $N \geq 2, \mu$ is larger than $M+N=n$, which is the homogeneous dimension of $R^{n}$ in those points $(x, y) \in R^{M} \times R^{N}$ in which $x \neq 0$. Then we obtain $V=0$ a.e. in $\Omega$. However, $\mathscr{L}$ satisfies (3)-(5); hence, Definition 2.1 can be used, and our result applies.

\section{REgULARITY OF THE SOLUTIONS OF $\mathscr{L} u+V u=0$}

In the following we will denote

$$
D_{\mathscr{L}} u \equiv\left(X_{1} u, \ldots, X_{p} u\right)
$$

for every $u \in C_{0}^{\infty}(\Omega)$. We will call $H_{0}^{1}(\Omega)$ the closure of $C_{0}^{\infty}(\Omega)$ with respect to the norm

$$
\|u\|_{H^{1}(\Omega)}=\left(\int_{\Omega}|u|^{2}+\left|D_{L} u\right|^{2} d y\right)^{1 / 2},
$$

and we say that $u \in H_{\text {loc }}^{1}(\Omega)$ if $u \varphi \in H_{0}^{1}(\Omega)$ for every $\varphi \in C_{0}^{\infty}(\Omega)$. Let us now define what we mean by a solution. 
Definition 4.1. If $V$ is an $\mathscr{L}$-Morrey potential (or, more generally, an $\mathscr{L}$ Stummel-Kato potential), we say that $u \in H_{\mathrm{loc}}^{1}(\Omega)$ is a local weak solution of equation

$$
\mathscr{L} u+V u=0
$$

if and only if

$$
\int_{\Omega} D_{\mathscr{L}} u D_{\mathscr{L}} \varphi d x=\int_{\Omega} V(x) u(x) \varphi(x) d x \quad \forall \varphi \in C_{0}^{\infty}(\Omega) .
$$

Let us explicitly note that the integral in the right-hand side exists, by Remark 3.2 in [CGL] and Lemma 2.1 here. In [CGL] it is proved that any solution of (13) is continuous. We present here a slightly different proof, working on the level sets of $\Gamma$, instead of the balls of the metric. In this way we will obtain an estimate of the continuity modulus of $u$ in terms of $\Gamma$, from which the Hölder continuity of $u$ will follow when the potential $V \in L_{\mathscr{L}}^{1, \lambda}(\Omega)$. Indeed our results can be stated as follows.

Theorem 4.1. Assume that $V \in S(\Omega)$ and $U \subset \subset \Omega$. Then there exist constants $\alpha, C_{1}, r_{0}>0$ such that for any positive solution of $\mathscr{L} u+V u \geq 0$ on $U$ and any level set $\Omega_{r}\left(x_{0}\right)$ with $\Omega_{4 r}\left(x_{0}\right) \subset \subset U$ we have

$$
\left|u(x)-u\left(x_{0}\right)\right| \leq C_{1}\left[\Gamma\left(x, x_{0}\right)^{-\alpha} r^{-\alpha}+\frac{\eta(r)}{\left(\Gamma\left(x, x_{0}\right) r\right)^{\alpha / 2}}+\eta\left(\frac{r^{1 / 2}}{\Gamma\left(x, x_{0}\right)}\right)\right] \sup _{\Omega_{r}\left(x_{0}\right)}|u|
$$

for almost all $x \in \Omega_{r}\left(x_{0}\right)$.

Corollary 4.1. Assume that $V \in L_{\mathscr{L}}^{1, \lambda}(\Omega)$ and $U \subset \subset \Omega$. Then there exist constants $\alpha, C_{1}, r_{0}>0$ such that for any positive solution $u$ of $\mathscr{L} u+V u \geq 0$ on $U$ and any level set $\Omega_{r}\left(x_{0}\right)$ with $\Omega_{r}\left(x_{0}\right) \subset \subset \Omega$ we have

$$
\begin{aligned}
&\left|u(x)-u\left(x_{0}\right)\right| \leq C_{1}\left[\Gamma\left(x, x_{0}\right)^{-\alpha} r^{-\alpha}+r^{\lambda-1}\left(\Gamma\left(x, x_{0}\right)^{\alpha / 2} r^{-\alpha / 2}\right.\right. \\
&+\left.\left.+\Gamma\left(x, x_{0}\right)^{-(\lambda-1) / 2} r^{-(\lambda-1) / 2}\right)\right] \sup |u|
\end{aligned}
$$

for almost all $x \in \Omega_{r}\left(x_{0}\right)$.

Remark 4.1. From the estimates (11) and (12) we get

$$
\Gamma\left(x, x_{0}\right)^{-1} \leq C \frac{|B(x, d(x, y))|}{d(x, y)^{2}} \leq C r^{Q\left(x_{0}\right)-2} .
$$

Then Corollary 4.1 implies that $u$ is Hölder continuous.

Remark 4.2. When $\mathscr{L}$ is a subelliptic laplacian and has constant homogeneous dimension $Q$, Corollary 4.1 simply reduces to: For any positive solution $u$ of $\mathscr{L} u+V u \geq 0$ on $U$ and any ball $\overline{B\left(x_{0}, 4 r\right)} \subset \subset U$ we have

$$
\begin{aligned}
\left|u(x)-u\left(x_{0}\right)\right| \leq & C_{1}\left\{\left(\frac{d\left(x, x_{0}\right)}{r}\right)^{\alpha(Q-1) / 2}\right. \\
& \left.+r^{\lambda-1}\left[\left(\frac{d\left(x, x_{0}\right)}{r}\right)^{\alpha(Q-1) / 2}+\left(\frac{d\left(x, x_{0}\right)}{r}\right)^{(\lambda-1)(Q-1) / 2}\right]\right\} \sup |u|
\end{aligned}
$$

for every $x \in B\left(x_{0}, r\right)$. 
Let us begin with some lemmas. If $\Lambda$ is the function defined in $\S 3$, we define

$$
E(x, r)=\Lambda(x, r) / r^{2}, \quad x \in R^{n}, r>0 .
$$

For $x_{0} \in \Omega, E\left(x_{0}, \cdot\right)$ is a polynomial with positive coefficients; hence, it is increasing, and we will denote its inverse by $F\left(x_{0}, \cdot\right)$. Now, from (10) it is possible to deduce that

Proposition 4.1. For every bounded set $U \subset \subset \Omega$ there exist constants $C_{0}, C_{1}$, $C_{2}, r_{0}$ such that for every $x \in U, 0<r<r_{0}$ we have

$$
\begin{aligned}
B\left(x, C_{1} F(x, r)\right) & \subseteq \Omega_{r}(x) \subseteq B\left(x, C_{2} F(x, r)\right), \\
\left|\Omega_{R}\left(x_{0}\right)\right| & \leq C_{0} R F^{2}\left(x_{0}, R\right)
\end{aligned}
$$

(see [CGL, Appendix $\mathrm{A} 1$ and A2] for a detailed proof).

Lemma 4.1 (existence of a cut-off function). For every bounded set $U \subset \subset \Omega$ for every $x_{0} \in U$, for every $r, \varrho>0$ with $r<\varrho$ and $\Omega_{e}\left(x_{0}\right) \subseteq U$, there exists $a$ cut-off function $\psi \in C_{0}^{\infty}\left(\Omega_{\ell}\left(x_{0}\right)\right)$ such that $0 \leq \psi \leq 1$ in $\Omega_{\ell}\left(x_{0}\right), \psi \equiv 1$ in $\Omega_{r}\left(x_{0}\right)$, and $\left|D_{\mathscr{L}} \psi\right| \leq 2 \varrho /(\varrho-r) d\left(y, x_{0}\right)$.

Proof. Let $\xi \in C_{0}^{\infty}([0, \varrho])$ be such that $\xi \equiv 1$ on $[0, r], 0 \leq \xi \leq 1$ on $[0, t]$, and $\left|\xi^{\prime}\right| \leq 2 / \varrho-r$. The function $\psi(y)=\xi\left(1 / \Gamma\left(y, x_{0}\right)\right)$ satisfies all the previous conditions.

Lemma 4.2 (Caccioppoli type inequality). Let us suppose that $V \in S(\Omega)$. Suppose that $u \in H_{\text {loc }}^{1}(\Omega), u \geq 0$ and satisfies $\mathscr{L} u+V u=0$ in $\Omega$. Then for every bounded set $U \subset \subset \Omega$ there exists $C_{1}, \varrho_{0}>0$ such that for every $\varrho<\varrho_{0}$, with $\overline{\Omega_{4 e}(x)} \subset U$, we have

$$
\int_{\Omega_{\ell}(x)}\left|D_{\mathscr{L}} u\right|^{2} d y \leq C_{1} \frac{\varrho}{\left|\Omega_{2 \varrho}(x)\right|} \int_{\Omega_{2 \ell}(x)} u^{2} d y .
$$

Proof. In Theorem 3.7 in [CGL] the same result is proved on the balls of the metric. Hence, using Proposition 4.2 and the existence of a cut-off function we easily get the estimate.

Proposition 4.2 (see [CGL]). For every $u \in C_{0}^{\infty}(\Omega)$ a.e. $x \in \Omega$ and every $r>0$ such that $\operatorname{supp}(u) \subset \subset \Omega_{r}(x)$

$$
u(x)=-\int_{\Omega_{r}(x)} L u(y) \Gamma(x, y) d y .
$$

Proof of Theorem 4.1. Let us denote by $\varphi$ a cut-off function of class $C_{0}^{\infty}\left(\Omega_{2 r}\left(x_{0}\right)\right)$ such that $0 \leq \varphi \leq 1, \varphi \equiv 1$ on $\Omega_{3 / 2 r}\left(x_{0}\right)$, and

Then

$$
\left|D_{H} \varphi\left(x_{0}\right)\right| \leq C_{1} \frac{1}{d\left(x, x_{0}\right)}
$$

$$
\begin{aligned}
u(x)-u\left(x_{0}\right)= & -\int\left(\Gamma(x, y)-\Gamma\left(x_{0}, y\right)\right) V(y) \varphi(y) u(y) d y \\
& +\int\left(\Gamma(x, y)-\Gamma\left(x_{0}, y\right)\right) D_{H} \varphi(y) D_{H} u(y) d y \\
& -\int\left(D_{H} \Gamma(x, y)-D_{H} \Gamma\left(x_{0}, y\right)\right) D_{H} \varphi(y) u(y) d y \\
= & \text { I }+ \text { II }+ \text { III. }
\end{aligned}
$$


Now,

$$
\left|\Gamma(x, y)-\Gamma\left(x_{0}, y\right)\right| \leq C\left(\frac{1}{\Gamma\left(x_{0}, x\right) r}\right)^{\alpha} \Gamma\left(x_{0}, y\right)
$$

hence, II can be estimated as

$$
\mathrm{II} \leq C\left(\frac{1}{\Gamma\left(x_{0}, x\right) r}\right)^{\alpha} \int_{\Omega_{2 r}\left(x_{0}\right) \backslash \Omega_{3 r / 2}\left(x_{0}\right)} \Gamma\left(x_{0}, y\right)\left|D_{H} u\right|\left|D_{H} \varphi\right| d x
$$

(by Cauchy-Schwartz inequality and the properties of the function $\varphi$ )

$$
\begin{aligned}
\leq & \frac{1}{r}\left(\frac{1}{\Gamma\left(x_{0}, x\right) r}\right)^{\alpha}\left(\int_{\Omega_{2 r}\left(x_{0}\right) \backslash \Omega_{3 r / 2}\left(x_{0}\right)}\left|D_{H} u\right|^{2} d x\right)^{1 / 2} \\
& \times\left(\int_{\Omega_{2 r}\left(x_{0}\right) \backslash \Omega_{3 r / 2}\left(x_{0}\right)} \frac{1}{d^{2}\left(x, x_{0}\right)} d x\right)^{1 / 2}
\end{aligned}
$$

(by the Caccioppoli inequality and Proposition 4.2)

$$
\begin{aligned}
& \leq \frac{1}{r}\left(\frac{1}{\Gamma\left(x_{0}, x\right) r}\right)^{\alpha}\left(\frac{r}{\Omega_{2 r}\left(x_{0}\right)} \int_{\Omega_{2 r}\left(x_{0}\right)} u^{2} d x\right)^{1 / 2}\left(\frac{\left|\Omega_{2 r}\left(x_{0}\right)\right|}{F^{2}\left(x_{0}, r\right)}\right)^{1 / 2} \\
& \leq\left(\frac{1}{\Gamma\left(x_{0}, x\right) r}\right)^{\alpha} \sup |u| .
\end{aligned}
$$

Analogously

$$
\mathrm{III} \leq\left(\frac{1}{\Gamma\left(x_{0}, x\right) r}\right)^{\alpha} \sup |u|
$$

Let us finally estimate I:

$$
\begin{aligned}
I= & \int_{1 / \Gamma\left(y, x_{0}\right)>N / \Gamma\left(y, x_{0}\right)}\left(\Gamma(x, y)-\Gamma\left(x_{0}, y\right)\right) V(y) \varphi(y) u(y) d y \\
& +\int_{1 / \Gamma\left(y, x_{0}\right) \leq N / \Gamma\left(y, x_{0}\right)}\left(\Gamma(x, y)-\Gamma\left(x_{0}, y\right)\right) V(y) \varphi(y) u(y) d y \\
= & A+B .
\end{aligned}
$$

Now,

$$
\begin{aligned}
A & \leq \frac{1}{N^{\alpha}} \int_{1 / \Gamma\left(x_{0}, y\right) \leq 2 r} \Gamma\left(x_{0}, y\right) V(y) \sup |u| \leq \frac{1}{N^{\alpha}} \eta(r) \sup |u| \\
& \leq \frac{\eta(r)}{\left(r \Gamma\left(x, x_{0}\right)\right)^{\alpha / 2}} \sup |u|
\end{aligned}
$$

if we choose $N=\left(r \Gamma\left(x, x_{0}\right)\right)^{1 / 2}$.

Since

$$
\frac{1}{\Gamma(x, y)} \leq C\left(\frac{1}{\Gamma\left(x, x_{0}\right)}+\frac{1}{\Gamma\left(x_{0}, y\right)}\right) \leq C(N+1) \frac{1}{\Gamma\left(x, x_{0}\right)},
$$


$B$ can be estimated as

$$
\begin{aligned}
B & \leq \int_{1 / \Gamma(y, x) \leq C(N+1) / \Gamma\left(y, x_{0}\right)} \Gamma(x, y) V(y) u(y) d y \\
& \leq \int_{1 / \Gamma\left(y, x_{0}\right) \leq N / \Gamma\left(y, x_{0}\right)} \Gamma\left(x_{0}, y\right) V(y) u(y) d y \\
& \leq \sup |u| \eta\left((N+1) \frac{1}{\Gamma\left(y, x_{0}\right)}\right)=C_{1} \sup |u| \eta\left(2\left[\frac{r}{\Gamma\left(y, x_{0}\right)}\right]\right) .
\end{aligned}
$$

\section{REFERENCES}

[AS] M. Aizenman and B. Simon, Brownian motion and Harnack's inequality for Schrödinger equations, Comm. Pure Appl. Math. 35 (1982), 209-271.

[CGL] G. Citti, N. Garofalo, and E. Lanconelli, Harnack's inequality for sum of squares of vector fields plus a potential, preprint.

[CFG] F. Chiarenza, E. Fabes, and N. Garofalo, Harnack's inequality for Schrödinger operator and the continuity of solutions, Proc. Amer. Math. Soc. 98 (1986), 415-425.

[D] G. Di Fazio, Hölder continuity of solutions for some Schrödinger equations, Rend. Sem. Mat. Univ. Padova 79 (1988), 173-183.

[F] G. B. Folland, A fundamental solution for a subelliptic operator, Bull. Amer. Math. Soc. 79 (1973), 373-376.

[HK] A. Hinz and H. Kalf, Subsolutions estimates and Harnack's inequality for Schrödinger operator, J. Reine Angew. Math. 404 (1990), 118-134.

[LU] O. Ladyzhenskaya and O. Uraltseva, Linear and quasilinear elliptic equations, Academic Press, New York, 1968.

[NSW] A. Nagel, E. M. Stein, and S. Wainger, Balls and metrics defined by vector fields. I: Basic properties, Acta Math. 155 (1985), 103-147.

[SC] A. Sanchez-Calle, Fundamental solutions and geometry of sum of squares of vector fields, Invent. Math. 78 (1984), 143-160.

[Si] C. G. Simander, An elementary proof of Harnack's inequality for Schrödinger operators and related topics, Math. Z. 203 (1990), 129-152.

[S] G. Stampacchia, Le problème de Dirichelet pour les équations elliptiques au second ordre à coefficients discontinus, Ann. Inst. Fourier (Grenoble) 15 (1965), 189-256.

Universitá di Bologna, Piazza di Porta S. Donato 5, Bologna, Italy

E-mail address: Citti@dm.unibo.it

Universitá di Catania, Viale A. Doria 6, Catania, Italy

E-mail address: DiFazio@mathct.cineca.it 